\title{
All-Optical Reversible Logic Gates with Optically Controlled Bacteriorhodopsin Protein-Coated Microresonators
}

\author{
Sukhdev Roy, ${ }^{1}$ Purnima Sethi, ${ }^{1}$ Juraj Topolancik, ${ }^{2,3}$ and Frank Vollmer ${ }^{2,4,5}$ \\ ${ }^{1}$ Department of Physics and Computer Science, Dayalbagh Educational Institute, Dayalbagh, Agra 282 110, India \\ ${ }^{2}$ Biofunctional Photonics Group, The Rowland Institute, Harvard University, 100 Edwin H. Land Boulevard, Cambridge, \\ MA 02142, USA \\ ${ }^{3}$ Department of Electrical and Computer Engineering, Northeastern University, 360 Huntington Avenue, Boston, \\ MA 02115, USA \\ ${ }^{4}$ Wyss Institute for Biologically Inspired Engineering, Harvard University, HIM, 10th Floor, 4 Blackfan Circle, Boston, \\ MA 02115, USA \\ ${ }^{5}$ Laboratory of Biophotonics and Biosensing, Max Planck Institute for the Science of Light, 91058 Erlangen, Germany
}

Correspondence should be addressed to Sukhdev Roy, sukhdevroy@dei.ac.in

Received 15 April 2011; Accepted 3 June 2011

Academic Editor: Ken-Tye Yong

Copyright () 2012 Sukhdev Roy et al. This is an open access article distributed under the Creative Commons Attribution License, which permits unrestricted use, distribution, and reproduction in any medium, provided the original work is properly cited.

We present designs of all-optical reversible gates, namely, Feynman, Toffoli, Peres, and Feynman double gates, with optically controlled microresonators. To demonstrate the applicability, a bacteriorhodopsin protein-coated silica microcavity in contact between two tapered single-mode fibers has been used as an all-optical switch. Low-power control signals $(<200 \mu \mathrm{W})$ at $532 \mathrm{~nm}$ and at $405 \mathrm{~nm}$ control the conformational states of the protein to switch a near infrared signal laser beam at 1310 or $1550 \mathrm{~nm}$. This configuration has been used as a template to design four-port tunable resonant coupler logic gates. The proposed designs are general and can be implemented in both fiber-optic and integrated-optic formats and with any other coated photosensitive material. Advantages of directed logic, high Q-factor, tunability, compactness, low-power control signals, high fan-out, and flexibility of cascading switches in 2D/3D architectures to form circuits make the designs promising for practical applications.

\section{Introduction}

There is tremendous research effort to achieve all-optical information processing for ultrafast and ultrahigh bandwidth communication and computing. The natural parallelism of optics along with advances in fabricating microand nanostructures has opened up exciting possibilities to generate, manipulate, and detect light and to tailor the optical molecular response for low-power all-optical computing [1-5].

A switch is the basic building block of information processing systems and optical logic gates are integral components of higher optical computing circuits. Conventional classical computing is based on Boolean logic that is irreversible, that is, the inputs cannot be inferred from the output, as the number of output bits is less than the inputs. This leads to destruction of information and hence to the dissipation of a large amount of energy [6-9].
Conservative and reversible logic circumvents this problem by having equal number of inputs and outputs and opening up the possibility of ultra-low power computing [6-9]. It is also compatible with revolutionary optical and quantum computing paradigms. Quantum arithmetic has to be built from reversible logical components, as unitary operations are reversible and hence quantum networks effecting elementary arithmetic operations such as addition, multiplication, and exponentiation cannot be directly deduced from their classical Boolean counterparts [10].

Several reversible logic gates have been proposed that include Fredkin gate (FG), Feynman gate, Toffoli gate (TG), Peres gate, and Feynman double gate [10-12]. A gate is conservative if the Hamming weight of its input equals the Hamming weight of its output. A number of physical implementations for the universal Fredkin gate have been proposed based on nonlinear optical bistable elements [13], 
interferometers $[9,14,15]$, linear optics $[16,17]$, complementary metal-oxide semiconductor (CMOS), cellular automata [18], silicon-on-insulator (SOI) [19], cavity quantum electrodynamics (QED) [20], spin states [21], recombination enzymes, and DNA membranes [22]. All-optical implementation of Fredkin gate has also been demonstrated experimentally with semiconductor optical amplifiers (SOA) [23] and recently with optical fiber-based nonlinear optical loop mirror (NOLM) [24].

However, there have been fewer designs proposed to implement other reversible logic gates. Recently, a photophysical design of a molecular Feynman gate has been proposed using fluorophores as molecular switches [25] and the quantum Tofolli gate with linear optics [26], atom-cavity interaction [27], and trapped ions [28].

The major challenge in the practical realization of optical logic has so far been in meeting essential requirements of cascadability, fan-out, logic-level restoration, input-output isolation, absence of critical level biasing, logic level independent of loss and of course low-power operation [3]. To effectively compete with future advancements in electronic implementations, optical logic has to be achieved at ultralow power levels. However, advancements in fabricating nanostructures (resonators and plasmonics), quantum dots, single molecule, and single photon devices, in even silicon, offer exciting possibilities to achieve this objective [1-5].

Application of the extremely sensitive and versatile microresonator structures for switching and computing applications has evoked tremendous interest due to their ultra-high $Q$-factor, low switching threshold, tunability, and ultra compactness [29-34]. A very wide range of microcavity shapes can be fabricated that include Fabry-Perot cavities, spheres, cylinders, disks, torroids, 1D/2D photonic crystals, and large-scale arrays of ultrahigh- $Q$ coupled nanocavities in photonic crystals [29]. They have also been shown to be feasible for building optical microcavity network on a chip for applications in quantum information processing [35].

Silica microcavities have inherent advantages of ultrahigh Q-factor $\left(\sim 10^{9}\right)$, relatively simple fabrication, small mode volume, near lossless fiber coupling, possibility of on-chip integration, and control of the coupling efficiency through taper by the change of the fiber thickness [29, $36,37]$. The recent demonstration of a fiber-taper-coupled ultrahigh-Q microtoroid on a chip enables integration of wafer-based functions with ultralow-loss fiber-coupled quantum devices. The bulk optical loss from silica is also exceptionally low, and record $Q$ factors of $8 \times 10^{9}$ (and finesse of $2.3 \times 10^{6}$ ) have been reported [29, 36, 37]. Ultrahigh-Q microtoroidal and bottleneck silica resonators represent a distinct class of optical microresonators with Q's in excess of $10^{8}$ and a simple and customizable mode structure enabling full tunability $[36,37]$. Due to the high $Q$-factor and the small dimensions, switching at low-power is feasible. In addition to this, coating the microcavity with a photosensitive material can further lead to switching at ultralow powers [38].

Silica microsphere optical resonators coated with a conjugated polymer [39] or silicon-nanocrystals [40] have been reported, in which optically induced thermo-optic switching takes place. In the case of conjugated polymer, $Q \sim 1.3 \times 10^{8}$ and a $405 \mathrm{~nm}$ pump at $4.9 \mu \mathrm{W}$ result in a switching on/off time of $\sim 50 \mathrm{~ms}$ [39]. On the other hand, silicon-nanocrystal-coated resonator $Q \sim 1.3 \times 10^{5}$ and a $488 \mathrm{~nm}$ pump at $3.4 \mu \mathrm{W}$ results in a switching on-off time of $\sim 25-30 \mathrm{~ms}$ [40]. However, it would be better to have a sensitive photochromic material-coated resonator for alloptical switching applications at low powers.

The photochromic protein bacteriorhodopsin (BR), which is found in the purple membrane of Halobacterium halobium, has emerged as an excellent material for biomolecular photonic applications due to its unique advantages [41-43]. It exhibits high quantum efficiency of converting light into a state change, large absorption cross-section and nonlinearities, robustness to degeneration by environmental perturbations, high stability towards photodegradation and temperature, response in the visible spectrum, low production cost, environmental friendliness, capability to form thin films in polymers and gels, and flexibility to tune its kinetic and spectral properties by genetic engineering techniques, for device applications [42-44]. A wide range of applications has been proposed for BR. Its properties of proton pumping and photoelectricity have been used for instance in desalination of seawater, conversion of sunlight to electricity, ultrafast light detection, chemo- and biosensing, artificial retinas, photon counters, and photovoltaic converters. Its photochromic property has resulted in applications that include pattern recognition systems, information recording, associative and 3D memories, holography, second harmonic generation, saturable absorption, wave mixing and phase conjugation, nonlinear optical filtering, optical bistability, mode locking, spatial light modulation, optical image processing, logic gates, neural networks, incoherent to coherent conversion, optical displays, beam deflection, and even for generation of slow and fast light [42-46].

The BR molecule initially excited by photons undergoes several structural transformations in a complex photocycle $\mathrm{B}_{570} \rightarrow \mathrm{J}_{625} \rightarrow \mathrm{K}_{610} \rightarrow \mathrm{L}_{550} \rightarrow \mathrm{M}_{410} \rightarrow \mathrm{N}_{520} \rightarrow \mathrm{O}_{640} \rightarrow$ $\mathrm{B}_{570}$ that generates intermediate states spanning the entire visible region of the spectrum where the subscripts denote the peak absorption wavelengths. An important feature of all the intermediates is their ability to be photochemically switched back to the initial B state by shining light at a wavelength that corresponds to the absorption peak of the intermediate in question. Genetic mutations of BR through biotechnological procedures such as variation in the polymer environment including $\mathrm{pH}$, temperature, degree of hydration, and addition of chemicals can alter both the kinetic and spectral properties of BR. Replacement of retinal by analog structures also has a strong influence on the photochemical cycle, and the absorption spectrum can be changed to the desired wavelength regions [42-46].

Recently, all-optical switching in the near-infrared with the ultra-sensitive bacteriorhodopsin (BR) protein-coated silica microcavities has been reported [47]. The state of the $\mathrm{BR}$ in optical microcavities is controlled by a lowpower $(<200 \mu \mathrm{W})$ continuous green pump laser coupled to the microsphere cavity using a tapered fiber [47, 48]. We have used this basic switch configuration to design 


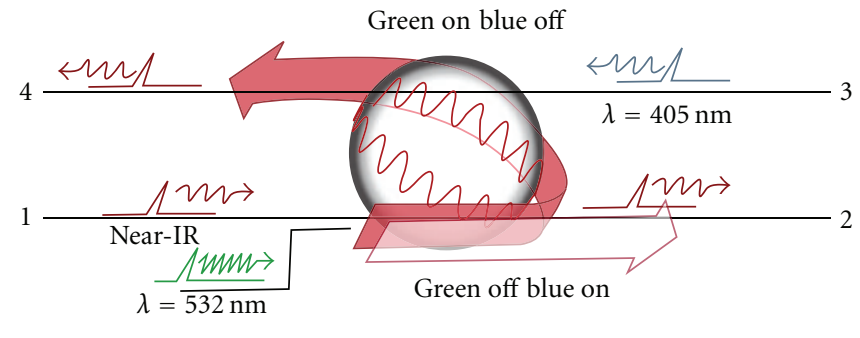

(a)

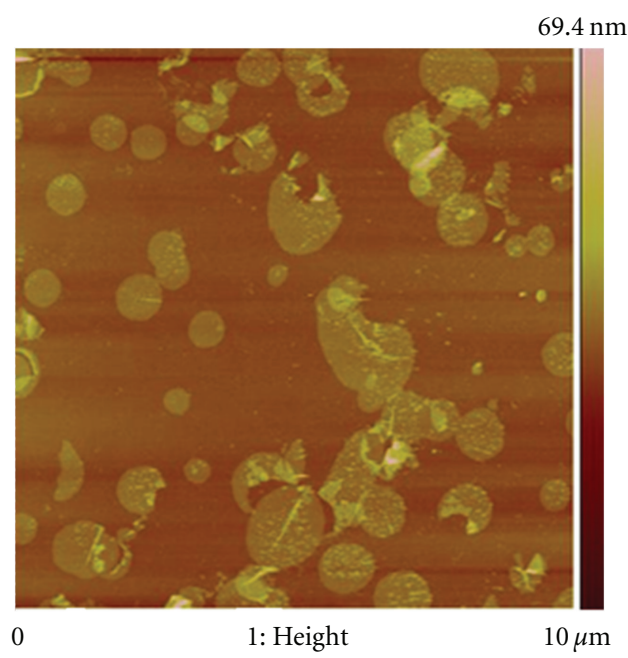

(b)

FIGURE 1: (a) Schematic of the experimental set-up, (b) AFM image of bacteriorhodopsin molecules.

higher computing circuits such as half/full adder-subtractors, multiplexer/demultiplexer and arithmetic unit circuits [49]. We have also shown that it can be used to elegantly design the all-optical universal Fredkin logic gate [50].

The objective of this paper is to experimentally analyze this switching configuration in more detail, by measuring the variation in the resonant shift of the TE-resonant peak of the $1310 \mathrm{~nm}$ IR signal by alternate exposure to green $(532 \mathrm{~nm})$ and blue $(405 \mathrm{~nm})$ pump laser beams to control the BR conformational states for faster switching, and to present general all-optical designs of various other kinds of important reversible logic gates reported in the literature, namely, Feynman gate, Toffoli gate, Peres gate, and Feynman double gate, with optically controlled microresonators. To achieve this objective, we consider the BR-coated silica microresonator switch as a template. The proposed designs offer advantages of simple configuration, cascadability, large scale-integration, flexibility and low-power operation.

\section{Experimental Setup of BR-Coated Hybrid Molecular-Photonic Switch}

Figure 1(a) shows the schematic representation of a resonant coupler that can be used to demonstrate the all-optical switch with BR-coated microcavity. The tip of a single-mode fiber was melted in a butane nitrous oxide flame to form a $300 \mu \mathrm{m}$ diameter silica microsphere $\left(Q \sim 2 \times 10^{6}\right)$. Alternate electrostatic deposition of cationic poly(dimethyldiallyl) ammonium chloride (PDAC) and anionic BR membranes was used to adsorb three mono-layers of BR mutant D96N (Munich Innovative Biomaterials, Munich, Germany) onto the microsphere surface. A single oriented PDAC/BR monolayer $(\sim 55 \AA$ thick) was self-assembled onto the microcavity surface, in each cycle [47]. The microcavity $Q$ was slightly degraded to $\sim 5 \times 10^{5}$, by the BR adsorption process and the introduction of scattering impurities during each drying process. The AFM image of BR molecules is shown in Figure 1(b).

Two parallel single-mode fibers were tapered by hydrofluoric acid erosion and held $250 \mu \mathrm{m}$ apart in a standard $1 \mathrm{~cm}$ acid-resistant polystyrene cuvette. The BRcoated microsphere was then spring loaded between the two tapered fibers in air, after complete removal of the etchant. Resonant modes were excited with a distributed feedback (DFB) laser, operating around $1310 \mathrm{~nm}$, connected to Port 1 via single-mode fiber (SMF-28, Dow-Corning, Midland MI) through a fiber coupler. A green pump beam at $532 \mathrm{~nm}$ and a blue pump beam at $405 \mathrm{~nm}$ were injected into Port 1 and Port 3 of the switch, respectively. Two InGaAs photodiodes (PD1, PD2; PDA400 from Thorlabs) connected to fiber Ports 2 and 4 were used to monitor and record transmitted intensities of the near infrared probe beam with a LABVIEW program. To determine the resonance wavelength of the microsphere and changes thereof, the current of the DFB laser was swept with a sawtooth-shaped function every $\sim 200 \mathrm{~ms}$, thereby tuning the wavelength by $\sim 0.006 \mathrm{~nm} / \mathrm{mA}$. The resonance wavelength was then identified by determining the minimum (maximum) of the Lorentzian-shaped trough (peak) that appears in the transmission spectrum recorded at Port 2 (Port 4) on each wavelength sweep. For switching experiments, the wavelength of the DFB laser was fixed and slightly detuned from the resonance wavelength to allow for a maximum all-optical switching contrast.

\section{Results and Discussion}

3.1. All-Optical Switching in BR-Coated Microcavity. Photoexcitation of BR triggers a complex photocycle that involves isomerization and rotation of the retinal chromophore, and a proton transfer across the lipid membrane [41-44]. During the photocycle, the retinal changes its conformation between the all-trans, 15-antiprotonated Schiff base found in 


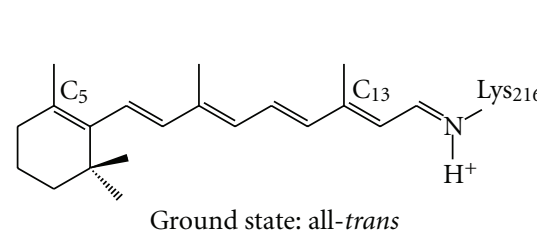

Ground state: all-trans

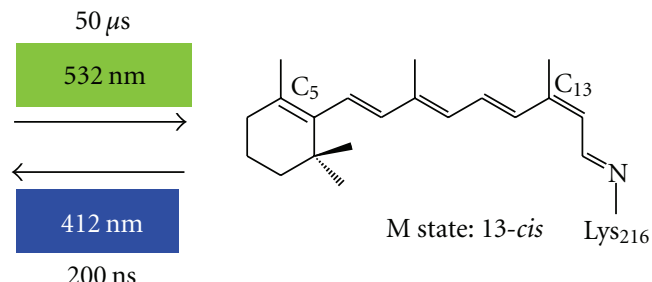

(a)

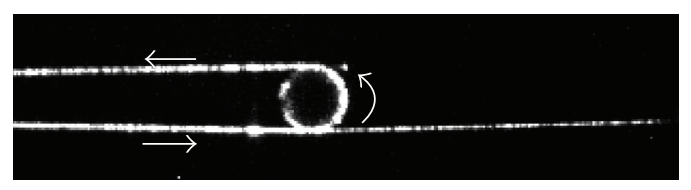

(b)

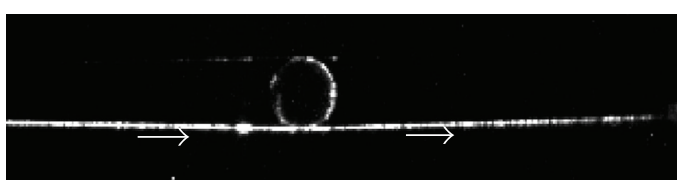

(c)

FIgURE 2: (a) Photo-induced molecular conformational switching between the ground and the M-intermediate states of BR, (b) IR image of $1310-\mathrm{nm}$ probe beam propagation with green pump beam on and blue off, and (c) IR-probe propagation with green pump off and blue on.

the thermodynamically stable ground state; 13-cis, 15-anti protonated Schiff base prevalent in its metastable M-state. These two states identify a bistable photochromic system used for light modulation. Excitation of the retinal protein around the ground state maximum absorption wavelength of $568 \mathrm{~nm}$ drives BR through several intermediaries into the $\mathrm{M}$-state within $50 \mu \mathrm{s}$. Conversely, illumination of BR around the M-state absorption peak of $412 \mathrm{~nm}$, directly recovers the ground state within $200 \mathrm{~ns}$ (Figure 2(a)). This photoreaction represents the molecular basis for all-optical switching with BR $[45,51]$.

In the BR-coated microcavity switch, the switching of the input signal operating at wavelength $1310 / 1550 \mathrm{~nm}$ between the output ports ( 2 and 4 ) is photoinduced with a fibercoupled green pump laser (at $532 \mathrm{~nm}$ ) which controls the conformational state of the adsorbed BR. Photoinduced isomerization of the retinal alters the optical properties of the BR membrane such as the absorption spectrum, the molecular polarizability, and the hyperpolarizabilities. Hence, the conformational change perturbs the optical modes interacting with $\mathrm{BR}$ even when there is little or no spectral overlap between the mode frequency and the BR electronic transition bands. Resonant modes in the optical microcavity significantly enhance this interaction conveying this perturbation as a frequency shift of optical resonances.

The molecularly functionalized microcavity thus redirects the flow of near-infrared light beam between two optical fibers. With the pump OFF, the probing light from input Port 1 is detuned from resonance and is directly transmitted into the output Port 2. The pump evanescently excites whispering gallery modes (WGM) propagating around the microsphere's equator, inducing photoisomerization along their path. A low green cw laser $(<200 \mu \mathrm{W}$ at $532 \mathrm{~nm})$ is sufficient for this purpose, as its effective absorption is resonantly enhanced. Isomerization reduces the retinal polarizability, tuning the resonance wavelength of the microsphere to match the wavelength of the infrared probe which is then rerouted from input Port 1 into the output Port 4 . The transmission spectra exhibited an extinction of $-9.4 \mathrm{~dB}$ in Port

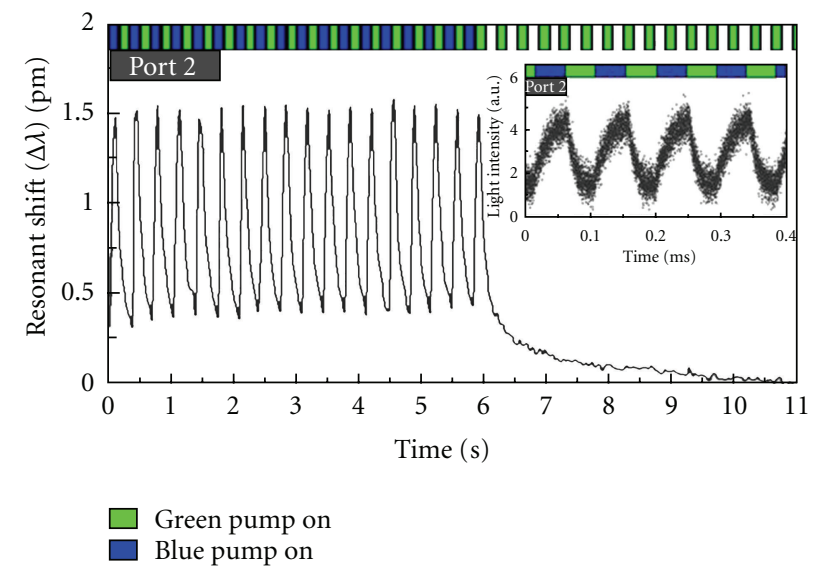

FIGURE 3: Variation in the resonant shift of the TE-resonant peak of the $1310 \mathrm{~nm}$ IR signal by alternate exposure to green $(532 \mathrm{~nm})$ and blue $(405 \mathrm{~nm})$ pump beams. Inset shows the corresponding temporal switching response of a microsphere to a fast $10 \mathrm{kHz}$ modulation, when green and blue pump beams $(\sim 200 \mu \mathrm{W}, \mathrm{cw})$ were modulated with step functions at $\sim 0.1 \mathrm{~ms}$ intervals.

2 and a $9.8 \mathrm{~dB}$ increase in transmission in Port 4 [47-49]. Figure 2(b) shows the IR image of $1310 \mathrm{~nm}$ probe beam propagation with pump green beam on and blue turned off with light getting switched to Port 4 whereas Figure 2(c) with blue off and green on, it is transmitted to Port 2.

In this experiment, green $(532 \mathrm{~nm})$ and blue $(405 \mathrm{~nm})$ pump lasers, connected to Port 1 and 3, respectively, were used to control the conformation state of the retinal. Turning the green pump on and the blue off (13-cis isomer) routes the probe into output Port 4 . When the green pump is off and the blue is on (all-trans isomer), the near-IR probe $(1310 / 1550 \mathrm{~nm}$ telecom laser) is switched from Port 4 to output Port 2. The blue light beam $(405 \mathrm{~nm})$ helps in truncating the photocycle of the BR molecules at the Mintermediate state, which is near to its peak absorption wavelength of $412 \mathrm{~nm}$, which results in a faster switch-off. 


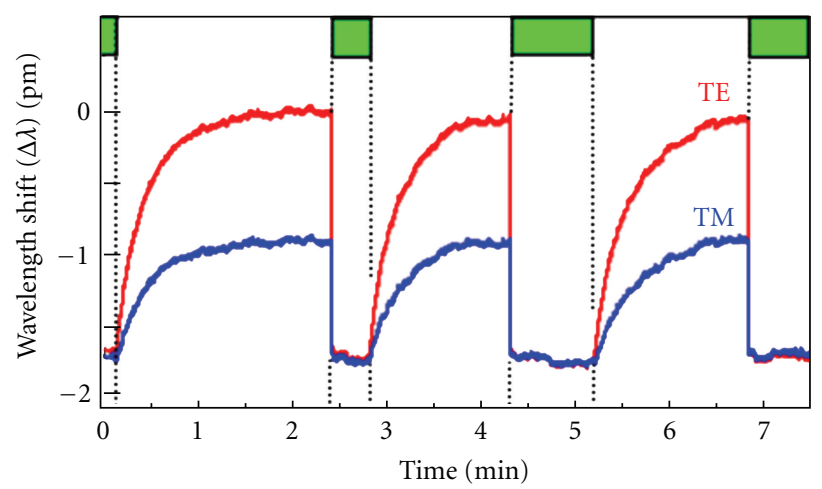

Green pump

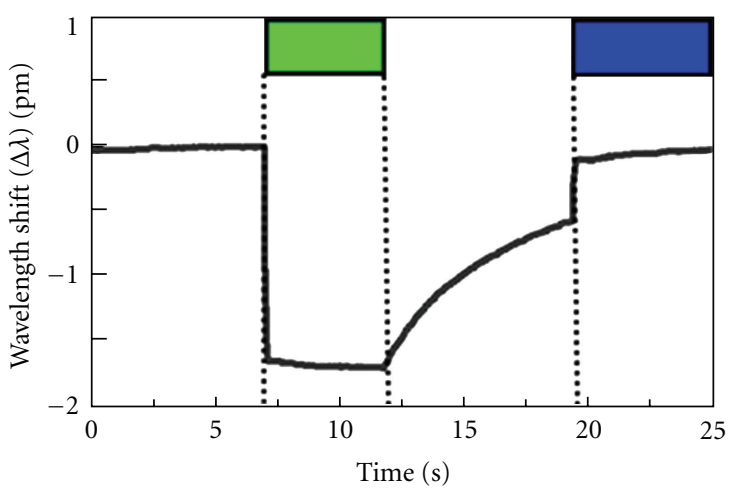

Green pump

Blue pump

(a)

(b)

FIGURE 4: (a) Dynamics of the ground-to-M state transformation observed by tracking wavelength shifts of TE- and TM-polarized microcavity modes on alternate exposure to green pump beam, (b) the slow thermal M-to-ground state relaxation turning into a fast photoreaction on exposure to the green pump along with the blue beam.

Figure 3 shows the variation in the resonant wavelength shift $(\Delta \lambda)$ of a transverse-electric- (TE-) polarized resonant mode on excitation with the modulating green and blue pump signals. It is evident that the alternating excitation with green and blue laser beams, results in the periodic shift in the resonant wavelength of about $1.2 \mathrm{pm}$. The inset shows the temporal response of a microsphere to a fast $10 \mathrm{kHz}$ modulation, when green and blue pump beams $(\sim 200 \mu \mathrm{W}, \mathrm{cw})$ were modulated with step functions at $\sim 0.1 \mathrm{~ms}$ intervals and the transmitted intensity of the probe beam was measured at Port 2. The IR-probe modulation was recorded with a fast scanning oscilloscope. The switch ON/OFF time $\mu$ s. Figure 4(a) depicts the dynamics of the ground-to-M state transformation observed by tracking wavelength shifts of TE- and TM-polarized microcavity modes on alternate exposure to green pump beam, and Figure 4(b) shows the slow thermal $\mathrm{M}$ to ground state relaxation turning into a fast photoinduced transition when the blue pump is applied at $t$ $\approx 19.5 \mathrm{~s}$.

For the low-pump intensities used in our measurements, the maximum modulation frequency was limited to $\sim 10 \mathrm{kHz}$, where considering the switching contrast to be defined here as the ratio of the signal change and the noise level, reduced to $\sim 2$. Enhanced switching contrast at higher modulation rates can be achieved with mode-locked green and blue pumps and higher optical densities. The switching speed of BR-based devices is limited by the timescale of the ground to M-state transition $\left(\tau_{568} \approx 50 \mu \mathrm{s}\right)$. D96N-BR is known to exhibit a much longer $\mathrm{M}$-state lifetime as compared to wildtype BR [42-46]. The speed of isomerization of the chromophore is also temperature dependent. It has been shown that the rate constant for M-state decay increases on increase in temperature [42-46]. An increase in temperature would result in faster switching of BR and hence the signal. The switching time can be further reduced by tailoring the BR intermediate lifetimes and/or phototransitions, by exploiting ultrafast photochromic transitions of the early BR intermediate states recently characterized and studied for switching $[52,53]$, or by coating the microcavity by other organic molecules such as diarylethenes that exhibit ultrafast photochromic transitions.

The ultra-high sensitivity of both microresonators and BR protein configuration results in switching at very low powers. At present, all-optical switching in BR-coated microcavity has not been optimized for practical applications. The energy/bit for switching that has been demonstrated corresponds to $\sim \mathrm{nJ} / \mathrm{bit}$. Both the minimum switching energy/bit and maximum switching speed need to be ascertained by exploiting the ultrafast initial intermediates. The $200 \mu \mathrm{W}$ switching power used in the experiment for the control signal can be further reduced by working with resonantly coupled coherent pump sources. This, however, would add another level of complexity to the control architecture. Working with the polarization states of $\mathrm{BR}$ can also add to the complexity or control [48]. The use of linearly polarized input light reduces the transmission at the off resonance frequency and increases the signal-to-noise ratio significantly. Using linearly polarized modes, the resonant wavelength shift ratio for the TE and TM polarizations is observed to be $2.1 \pm 0.1$, that is, the shift is about twice as large for TE as for the TM mode, which is due to retinal orientation in the BR membrane. Hence, TE modes have higher WGM peaks with higher intensity compared to TM modes.

Both green and blue pump beams can also be alternatively injected through a coupler at Port 1 . Hence, the silica microcavity in contact between two tapered fibers serves as a four-port tunable resonant coupler as shown in Figure 1(a).

3.2. Design of All-Optical Reversible Logic Gates. The alloptical BR-coated microcavity switch with a single control signal at $532 \mathrm{~nm}$ or in conjunction with a $405 \mathrm{~nm}$ can be used as a building block for designing all-optical reversible logic 


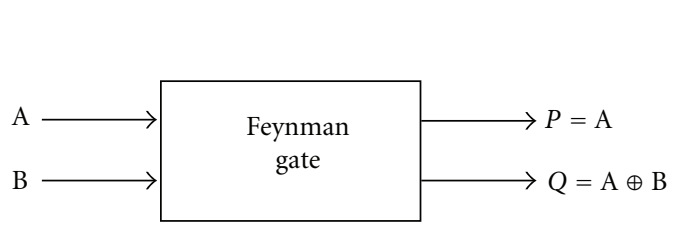

(a)

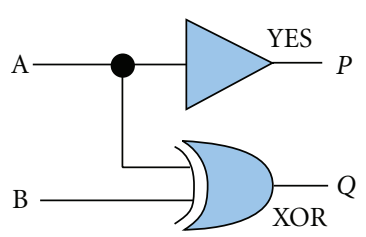

(b)

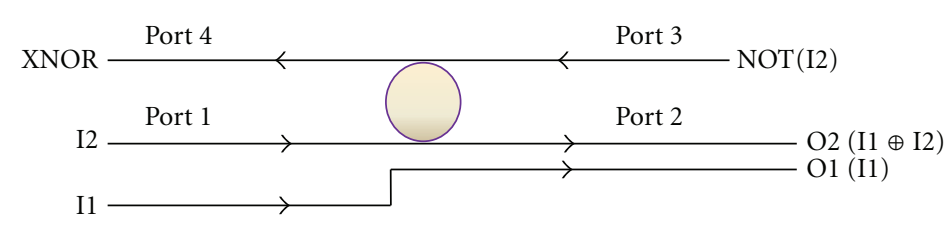

(c)

Figure 5: All-optical Feynman gate, (a) block diagram, (b) schematic of the logic circuit, and (c) schematic of the design using optically controlled microcavities. The dashed line shows the option of using an inverter circuit.

TABLE 1: Truth table of all-optical Feynman gate.

\begin{tabular}{|c|c|c|c|c|c|c|c|c|c|}
\hline \multicolumn{6}{|c|}{ Input } & \multicolumn{4}{|c|}{ Output } \\
\hline \multicolumn{2}{|c|}{$\mathrm{I} 1(\mu \mathrm{W})$} & \multicolumn{2}{|c|}{$\mathrm{I} 2(\mathrm{~mW})$} & \multicolumn{2}{|c|}{ NOT (I2) $(\mathrm{mW})$} & \multicolumn{2}{|c|}{$\mathrm{O} 1(\mu \mathrm{W})$} & \multicolumn{2}{|c|}{$\mathrm{O} 2(\mathrm{~mW})$} \\
\hline 0 & & 0 & & 1 & $(1.5)$ & 0 & & 0 & \\
\hline 0 & & 1 & $(1.5)$ & 0 & & 0 & & 1 & $(1.5)$ \\
\hline 1 & $(60)$ & 0 & & 1 & (1.5) & 1 & $(30)$ & 1 & (1.47) \\
\hline 1 & $(60)$ & 1 & $(1.5)$ & 0 & & 1 & (30) & 0 & $(0.03)$ \\
\hline
\end{tabular}

gates, namely, Feynman, Toffoli, Peres, and the Feynman double gates. We consider a combination of three inputs, namely, the IR signal at Port 1 and 3 and the control signal at Port 1 , and three outputs, that is, IR signal at Ports 2 and 4 and the control signal at Port 2.

3.2.1. All-Optical Feynman Gate. The Feynman gate is a $2 \times 2$ reversible gate shown schematically in Figure 5(a). It has 2 inputs and 2 outputs, with one input variable which is also the output. It can also be considered as an integration of a YES gate and an XOR gate, and is also known as controlledNOT gate (CNOT), Figure 5(b). The inputs and outputs are associated as $\mathrm{I} 1=\mathrm{O} 1$ and $\mathrm{O} 2=\mathrm{I} 1 \mathrm{XOR} \mathrm{I} 2$, as is also evident from the Truth Table 1.

The design of an all-optical Feynman gate using a BRcoated microcavity switch is as shown in Figure 5(c). We consider the inputs to be the control signal and the IR signal at Port 1, designated as inputs $\mathrm{I} 1$ and I2, respectively, while the control signal passing through the fiber coupler towards the output and the IR signal emerging at port 2 are considered as the two outputs, $\mathrm{O} 1$ and $\mathrm{O} 2$, respectively. The state of the device is initially off-resonance and the presence of the control input puts the device on resonance. We further consider the infrared input 12 to be fed into either Port 1 or Port 3 by two laser diodes one at a time, or possibly by a single diode at Port 1 connected to Port 3 , through an inverter.

Of the four input combinations, when no input is applied, the outputs are zero, due to the absence of any signal. When the control signal I1 is OFF, and the input I2 at Port 1 is $\mathrm{ON}$, it passes out to Port 2 due to the inactivation of the microcavity $(\mathrm{O} 2=$ High and $\mathrm{O} 1=\mathrm{I} 1=0)$. Since, input at Port
1 is ON, Port 3 is OFF and so there is also no output at Port 4. When, $\mathrm{I} 1$ is ON and $\mathrm{I} 2$ at Port 1 is OFF, the input NOT(I2) at Port 3 is $\mathrm{ON}$, which is switched by the microcavity to emerge at Port $2(\mathrm{O} 2=$ High and $\mathrm{O} 1=\mathrm{I} 1=1)$. Finally, when both $\mathrm{I} 1$ is $\mathrm{ON}$ and $\mathrm{I} 2$ at Port 1 is also ON, the signal I 2 is switched by the control signal to emerge at Port 4, leading to $\mathrm{O} 2=$ Low and $\mathrm{O} 1=\mathrm{I} 1=1$ ). Since, there is no input at Port 3 (it is OFF as Port 1 is ON), hence, it does not affect the outcome at Port 2.

We consider the experimental conditions, that is, a $200 \mu \mathrm{W}$ laser operating at $532 \mathrm{~nm}$ as the control signal at I1 and a $5 \mathrm{~mW}$ DFB laser operating at $1310 / 1550 \mathrm{~nm}$, which butt couples $\sim 30 \%$ light $(1.5 \mathrm{~mW})$ as the input signal at Ports 1 or 3. Considering our switching results [47], with $\sim 98 \%$ of light $(1.47 \mathrm{~mW})$ getting switched or routed from Port 1 to Port 4, or from Port 3 to Port 2, when the microcavity is activated, only $\sim 2 \%$ of the light then gets coupled into Port 2 or Port 4 , respectively $(0.03 \mathrm{~mW})$. Considering $50 \%$ power of the control signal coupled to the microcavity through a fiber coupler, the remaining power is considered to be detected at the output port that is, $\mathrm{O} 1(100 \mu \mathrm{W})$. In general, nearly lossless power transfer between optical fibers and high-Q optical resonators has been observed, and by proper selection of the taper diameter, negligible scattering loss takes place [54]. Considering positive logic and an upper limit of low output state to be $0.05 \mathrm{~mW}$ and a minimum threshold at $1.3 \mathrm{~mW}$ for the high state, for the IR output, and a corresponding upper limit of $5 \mu \mathrm{W}$ for the low state and a lower limit of $10 \mu \mathrm{W}$ for the high-state, for the green control beam, Table 1 results in the realization of all-optical Feynman gate. In addition to this, the output at Port 4 results in realization of XNOR logic operation. 


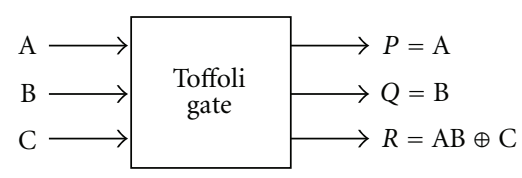

(a)

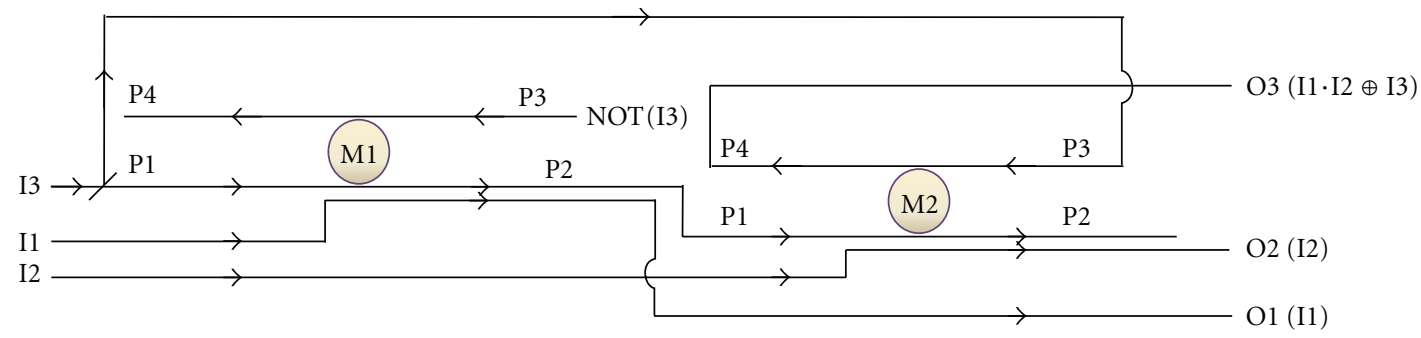

(b)

FIGURE 6: All-optical Toffoli gate, (a) block diagram, and (b) schematic of the design using optically controlled microcavities.

TABle 2: Truth table of all-optical Toffoli gate.

\begin{tabular}{|c|c|c|c|c|c|c|c|c|c|c|c|c|c|}
\hline \multicolumn{8}{|c|}{ Input } & \multicolumn{6}{|c|}{ Output } \\
\hline \multicolumn{2}{|c|}{$\mathrm{I} 1(\mu \mathrm{W})$} & \multicolumn{2}{|c|}{$\mathrm{I} 2(\mu \mathrm{W})$} & \multicolumn{2}{|c|}{ I3 $(\mathrm{mW})$} & \multicolumn{2}{|c|}{ NOT(I3) (mW) } & \multicolumn{2}{|c|}{$\mathrm{O} 1(\mu \mathrm{W})$} & \multicolumn{2}{|c|}{$\mathrm{O} 2(\mu \mathrm{W})$} & \multicolumn{2}{|c|}{$\mathrm{O} 3(\mathrm{~mW})$} \\
\hline 0 & & 0 & & 0 & & 1 & $(1.5)$ & 0 & & 0 & & 0 & \\
\hline 0 & & 0 & & 1 & (1.5) & 0 & & 0 & & 0 & & 1 & $(1.5)$ \\
\hline 0 & & 1 & (60) & 0 & & 1 & (1.5) & 0 & & 1 & $(30)$ & 0 & \\
\hline 0 & & 1 & $(60)$ & 1 & (1.5) & 0 & & 0 & & 1 & $(30)$ & 1 & $(1.5)$ \\
\hline 1 & $(60)$ & 0 & & 0 & & 1 & (1.5) & 1 & $(30)$ & 0 & & 0 & \\
\hline 1 & $(60)$ & 0 & & 1 & (1.5) & 0 & & 1 & (30) & 0 & & 1 & $(1.5)$ \\
\hline 1 & $(60)$ & 1 & $(60)$ & 0 & & 1 & (1.5) & 1 & (30) & 1 & $(30)$ & 1 & $(1.44)$ \\
\hline 1 & $(60)$ & 1 & $(60)$ & 1 & (1.5) & 0 & & 1 & (30) & 1 & (30) & 0 & $(0.03)$ \\
\hline
\end{tabular}

3.2.2. All-Optical Toffoli Gate. The Toffoli gate is a universal reversible logic gate, which is also known as the "controlledcontrolled-not" gate. It has a 3-bit input and output, and if the first two bits are set, it flips the third bit, as shown in Truth Table 2. It can be also described as mapping bits I1, I2, and I3 to I1, I2, and I3 XOR (I1 AND I2), as shown in Figure 6(a).

Considering the BR-coated microcavity switch described earlier, the design of an all-optical Toffoli gate is as shown in Figure 6(b), which is realized using two switches. We consider the two inputs as control signals I1 and I2, respectively, while the third input $\mathrm{I} 3$ is considered to be the input IR signal at Port P1 or at Port P3 of the first microcavity, by two laser diodes one at a time, or by a single diode at Port P1 connected to Port P3, through an inverter, (to make them out of phase), I3 is also connected directly to Port P3 of the second microcavity. Since two inputs in the Toffoli gate map out to two outputs, we have this implemented with the control signals I1 and I2 mapping out to $\mathrm{O} 1$ and $\mathrm{O} 2$, respectively. In this case, we consider an IR laser diode of $10 \mathrm{~mW}$ to butt couple $30 \%$ light of $3 \mathrm{~mW}$ to form input I3, which splits equally through the beam splitter to form the two inputs to the two microcavities, M1 and M2. This ensures consistency in the input signal intensities in all reversible logic gate designs and hence setting the same threshold values.
Considering the various possible combinations of the three inputs, as mentioned in Truth Table 2, for Case (i): When none of the inputs are applied, there are no outputs. Case (ii): When both I1 = I2 = 0 and I3 is applied, from P1, it emerges at P2 of M2, passing through P2 of M1 and P1 of M2. I3 which also inputs at P3 of M2 emerges out at P4 of $\mathrm{M} 2$ to $\mathrm{O} 3$, as both the microcavities are disabled. Case (iii): When $\mathrm{I} 1=0$ and $\mathrm{I} 2=1$ and $\mathrm{I} 3=0, \mathrm{O} 2=1$ and $\mathrm{O} 3=0$, as, although M2 is activated, no signal at P3 of M2 leads to P4 when $\mathrm{I} 1=0$ and $\mathrm{I} 2=1$ and $\mathrm{I} 3=0, \mathrm{P} 4$ of $\mathrm{M} 2=\mathrm{O} 3=0$. Case (iv): When $\mathrm{I} 1=0$ and $\mathrm{I} 2=1$ and $\mathrm{I} 3=1, \mathrm{O} 3$ is high as the signal is switched by M2 to P4 of M2. Input I3 at P3 of M2 also makes $\mathrm{P} 2$ of $\mathrm{M} 2$ high, although it is of no consequence in the Toffoli gate logic operation. Case (v): When I1 = 1 and $\mathrm{I} 2=0=\mathrm{I} 3, \mathrm{M} 1$ is activated and M2 is off, but with no signal $\mathrm{I} 3$ at $\mathrm{P} 1$ or $\mathrm{P} 3$ of $\mathrm{M} 2$, the output at $\mathrm{O} 3=0$. However, NOT(I3) which is high at P3 gets switched by M1 to emerge at P2 of M2. Case (vi): When I1 = 1, I2 = 0 and I3 = 1, M1 switches $\mathrm{I} 3$ to $\mathrm{P} 4$ and $\mathrm{I} 3$ at $\mathrm{P} 3$ of M2 passes over to P4 of M2 to result in $\mathrm{O} 3$ being high. Case (vii): When $\mathrm{I} 1=\mathrm{I} 2=1$ and $\mathrm{I} 3=0$, both microcavities are activated, but NOT(I3) is now high at $\mathrm{P} 3$, which gets switched by $\mathrm{M} 1$ and then M2 to result in a high output at $\mathrm{O} 3$. Case (viii): When $\mathrm{I} 1=\mathrm{I} 2=\mathrm{I} 3=1$, $\mathrm{I} 3$ gets switched by M1 to P4 and by M2 to its P2, to result in a low output at $\mathrm{O} 3$. The above combinations result in the circuit in 


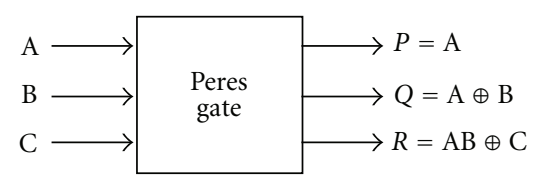

(a)

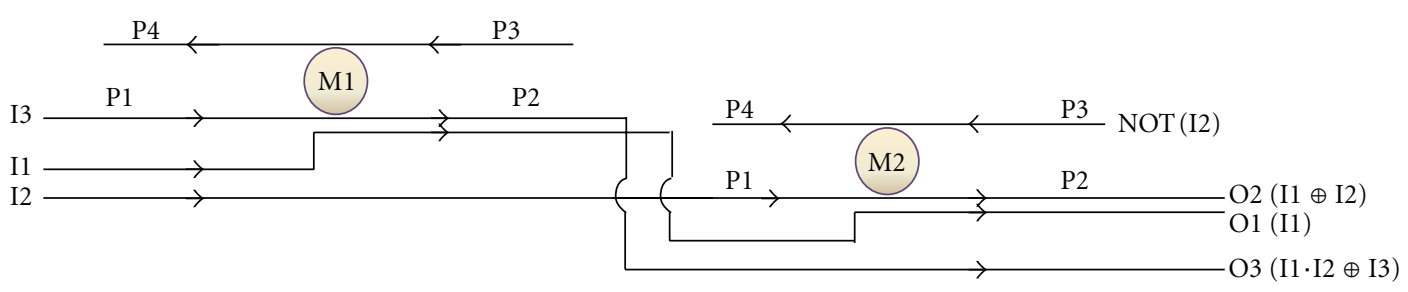

(b)

FIgURe 7: All-optical Peres gate (a) block diagram, (b) schematic of the design using optically controlled microcavities.

TABle 3: Truth table of all-optical Peres gate.

\begin{tabular}{|c|c|c|c|c|c|c|c|c|c|c|c|c|c|}
\hline \multicolumn{8}{|c|}{ Input } & \multicolumn{6}{|c|}{ Output } \\
\hline \multicolumn{2}{|c|}{$\mathrm{I} 1(\mu \mathrm{W})$} & \multicolumn{2}{|c|}{$\mathrm{I} 2(\mathrm{~mW})$} & \multicolumn{2}{|c|}{ NOT(I2) $(\mathrm{mW})$} & \multicolumn{2}{|c|}{$\mathrm{I} 3(\mathrm{~mW})$} & \multicolumn{2}{|c|}{$\mathrm{O} 1(\mu \mathrm{W})$} & \multicolumn{2}{|c|}{$\mathrm{O} 2(\mathrm{~mW})$} & \multicolumn{2}{|c|}{$\mathrm{O} 3(\mathrm{~mW})$} \\
\hline 0 & & 0 & & 1 & $(1.5)$ & 0 & & 0 & & 0 & & 0 & \\
\hline 0 & & 0 & & 1 & $(1.5)$ & 1 & (1.5) & 0 & & 0 & & 1 & $(1.5)$ \\
\hline 0 & & 1 & $(1.5)$ & 0 & & 0 & & 0 & & 1 & (1.5) & 0 & \\
\hline 0 & & 1 & $(1.5)$ & 0 & & 1 & (1.5) & 0 & & 1 & $(1.5)$ & 1 & $(1.5)$ \\
\hline 1 & $(60)$ & 0 & & 1 & $(1.5)$ & 0 & & 1 & (15) & 1 & $(1.47)$ & 0 & \\
\hline 1 & $(60)$ & 0 & & 1 & $(1.5)$ & 1 & (1.5) & 1 & (15) & 1 & $(1.47)$ & 0 & $(0.03)$ \\
\hline 1 & $(60)$ & 1 & $(1.5)$ & 0 & & 0 & & 1 & (15) & 0 & $(0.03)$ & 0 & \\
\hline 1 & $(60)$ & 1 & $(1.5)$ & 0 & & 1 & $(1.5)$ & 1 & $(15)$ & 0 & $(0.03)$ & 0 & $(0.03)$ \\
\hline
\end{tabular}

Figure 6(b), which implements Toffoli gate logic as shown in Truth Table 2.

3.2.3. All-Optical Peres Gate. The Peres gate is also a reversible $3 \times 3$ logic gate, with a schematic diagram shown in Figure $7(\mathrm{a})$. One output is the same as one input $(\mathrm{O} 1)$ whereas the second output is XOR of the first two inputs (O2) and the third output is XOR of the AND of first two inputs $(\mathrm{O} 3)$.

The design of an all-optical Peres gate with optically controlled microcavities is as shown in Figure 7(b), with the control signal I1 and the two IR signals I2 and I3 as the three inputs that result in the three outputs $\mathrm{O} 1, \mathrm{O} 2$, and $\mathrm{O} 3$, respectively. The control signal I1 activates both microcavities M1 and M2 in succession and maps to output O1.

Case (i): Initially, when none of the inputs are applied, only P3 of M2 is high, which passes over to P4 as M2 is not activated, resulting in all output ports being low. Case (ii): When $\mathrm{I} 1=\mathrm{I} 2=0$ and $\mathrm{I} 3=1$, both $\mathrm{M} 1$ and $\mathrm{M} 2$ are OFF and hence, $\mathrm{O} 2$ is low and $\mathrm{I} 3$ passes through to $\mathrm{O} 3$ and yields a high output. Case (iii): When $\mathrm{I} 1=0, \mathrm{I} 2=1$, and $\mathrm{I} 3=0$, again both microcavities are OFF, $\mathrm{I} 2$ passes over to $\mathrm{O} 2$ and makes it high and $\mathrm{O} 3$ is low. Case (iv): When $\mathrm{I} 1=0, \mathrm{I} 2=1$, and $\mathrm{I} 3=1, \mathrm{O} 2$, and $\mathrm{O} 3$ are both high as $\mathrm{I} 2$ and $\mathrm{I} 3$ get directly transmitted to these ports, without getting switched at M1 or M2. Case (v): When $\mathrm{I} 1=1$ and $\mathrm{I} 2=\mathrm{I} 3=0$, both $\mathrm{M} 1$ and
$\mathrm{M} 2$ are activated. Since $\mathrm{I} 3=0, \mathrm{O} 3$ is also low. $\mathrm{NOT}(\mathrm{I} 2)$ at P3 of M2 is high which gets switched by M2 to make O2 high. Case (vi): When $\mathrm{I} 1=1, \mathrm{I} 2=0$ and $\mathrm{I} 3=1$, again $\mathrm{O} 2$ is high due to switching of NOT(I2) by M2 to O2, whereas now, I3 gets switched by $\mathrm{M} 1$ to emerge at $\mathrm{P} 4$, making $\mathrm{O} 3$ again low. Case (vii): When $\mathrm{I} 1=\mathrm{I} 2=1$ and $\mathrm{I} 3=0, \mathrm{I} 2$ gets switched by $\mathrm{M} 2$ to its $\mathrm{P} 4$, leading to $\mathrm{O} 2$ getting low, while with no I3, $\mathrm{O} 3$ also gets low. Case (viii): When $\mathrm{I} 1=\mathrm{I} 2=\mathrm{I} 3=1$, I2 and I3 get switched by $\mathrm{M} 2$ and $\mathrm{M} 1$ respectively to result in $\mathrm{O} 2$ and $\mathrm{O} 3$ getting low. The various combinations of the inputs results in the desired outputs that satisfy Peres gate logic (Truth Table 3). The input I1 in terms of the control signal can also be fed in parallel to activate M1 and M2 through a beam splitter.

The various input-output combinations result in the realization of the Peres gate logic, as is evident from the Truth Table 3 .

3.2.4. All-Optical Feynman Double Gate. The Feynman Double gate is another $3 \times 3$ reversible gate which maps one input to one output, while the second output is the XOR of the first two inputs and the third output is the XOR of the second and third inputs, as shown in the block diagram in Figure 8(a).

Following the criterion applied earlier, the design based on two optically controlled microcavities is shown in Figure $8(\mathrm{~b})$. The input control signal I1 directly maps out 


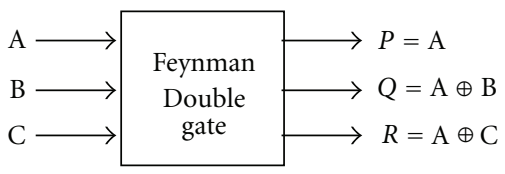

(a)

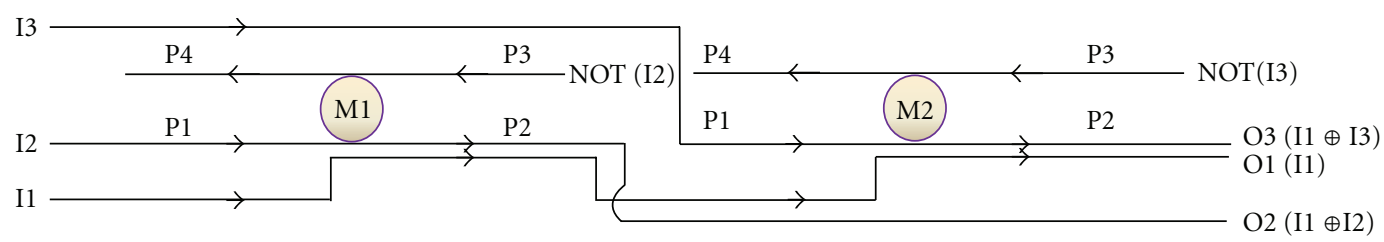

(b)

FIgURE 8: All-optical Feynman Double gate (a) block diagram, (b) schematic of the design using optically controlled microcavities.

TABle 4: Truth table of all-optical Feynman Double gate.

\begin{tabular}{|c|c|c|c|c|c|c|c|c|c|c|c|c|c|c|c|}
\hline \multicolumn{10}{|c|}{ Input } & \multicolumn{6}{|c|}{ Output } \\
\hline \multicolumn{2}{|c|}{$\mathrm{I} 1(\mu \mathrm{W})$} & \multicolumn{2}{|c|}{$\mathrm{I} 2(\mathrm{~mW})$} & \multicolumn{2}{|c|}{$\operatorname{NOT}(\mathrm{I} 2)(\mathrm{mW})$} & \multicolumn{2}{|c|}{$\mathrm{I} 3(\mathrm{~mW})$} & \multicolumn{2}{|c|}{$\operatorname{NOT}(\mathrm{I} 3)(\mathrm{mW})$} & \multicolumn{2}{|c|}{$\mathrm{O} 1(\mu \mathrm{W})$} & \multicolumn{2}{|c|}{$\mathrm{O} 2(\mathrm{~mW})$} & \multicolumn{2}{|c|}{$\mathrm{O} 3(\mathrm{~mW})$} \\
\hline 0 & & 0 & & 1 & $(1.5)$ & 0 & & 1 & $(1.5)$ & 0 & & 0 & & 0 & \\
\hline 0 & & 0 & & 1 & (1.5) & 1 & (1.5) & 0 & & 0 & & 0 & & 1 & (1.5) \\
\hline 0 & & 1 & (1.5) & 0 & & 0 & & 1 & (1.5) & 0 & & 1 & $(1.5)$ & 0 & \\
\hline 0 & & 1 & (1.5) & 0 & & 1 & (1.5) & 0 & & 0 & & 1 & $(1.5)$ & 1 & (1.5) \\
\hline 1 & $(60)$ & 0 & & 1 & (1.5) & 0 & & 1 & (1.5) & 1 & (15) & 1 & $(1.47)$ & 1 & (1.47) \\
\hline 1 & $(60)$ & 0 & & 1 & (1.5) & 1 & (1.5) & 0 & & 1 & (15) & 1 & $(1.47)$ & 0 & $(0.03)$ \\
\hline 1 & $(60)$ & 1 & (1.5) & 0 & & 0 & & 1 & (1.5) & 1 & (15) & 0 & $(0.03)$ & 1 & (1.47) \\
\hline 1 & $(60)$ & 1 & (1.5) & 0 & & 1 & (1.5) & 0 & & 1 & (15) & 0 & $(0.03)$ & 0 & $(0.03)$ \\
\hline
\end{tabular}

to output $\mathrm{O} 1$, satisfying the first requirement. The IR input signals I 2 and I 3 form the other two inputs, with outputs at $\mathrm{O} 2$ and $\mathrm{O} 3$. We consider the various combinations of the inputs resulting in respective outputs as described in Truth Table 4.

Case (i): When none of the inputs are applied, we get no outputs. Case (ii): When I1 = I2 = 0 and I $3=1$, both M1 and $\mathrm{M} 2$ are not activated and hence, $\mathrm{I} 3$ passes over to $\mathrm{O} 3$ to result in a high output. The NOT(I2) which is high, also does not get switched and passes over to P4 of M1. Case (iii): When $\mathrm{I} 1=0, \mathrm{I} 2=1$ and $\mathrm{I} 3=0$, in the absence of any switching by M1 and M2, I2 passes over to O2 to make it high and I3 ensures that $\mathrm{O} 3$ is low. Case (iv): When $\mathrm{I} 1=0, \mathrm{I} 2=\mathrm{I} 3=1$, since both $\mathrm{M} 1$ and $\mathrm{M} 2$ are $\mathrm{OFF}$, again $\mathrm{O} 2$ and $\mathrm{O} 3$ pass over to make $\mathrm{O} 2=\mathrm{O} 3=1$. Case $(\mathrm{v})$ : When $\mathrm{I} 1=1, \mathrm{I} 2=\mathrm{I} 3=0, \mathrm{M} 1$ and M2 are activated and NOT(12) and NOT(I3) are now high, which get switched at M1 and M2 respectively to make $\mathrm{O} 2$ and $\mathrm{O} 3$ high. Case (vi): When $\mathrm{I} 1=1, \mathrm{I} 2=0$ and $\mathrm{I} 3=$ 1 , NOT(I2) is high which is routed by M1 to O2, while I3 gets switched by $\mathrm{M} 2$ to emerge at $\mathrm{P} 4$ of $\mathrm{M} 2$ to result in a low output at O3. Case (vii): When I1 = I $2=1$ and I $3=0$, I2 gets switched by M1 to make O2 low and NOT(I3) gets switched by $\mathrm{M} 2$ to make $\mathrm{O} 3$ high. Case (viii): When $\mathrm{I} 1=\mathrm{I} 2=\mathrm{I} 3=1$, I 2 and $\mathrm{I} 3$ get switched by M1 and M2 to result in low outputs at $\mathrm{O} 2$ and $\mathrm{O} 3$, respectively.

The above combinations result in the circuit in Figure 8(b) that implements Feynman double gate logic as shown in Truth Table 4. This paper demonstrates the applicability of the concept of optically controlled microcavities coated with a photochromic material for all-optical information processing. The proposed designs of the various all-optical reversible logic gates have been realized considering positive logic and using common threshold values for the high and low-states, as defined in the case of Feynman gate.

$\mathrm{BR}$ is virtually transparent at near-infrared telecom band $(1310 / 1550 \mathrm{~nm})$ [41-46]. The tunable all-optical resonant coupler using a high Q-silica microcavity coated with ultrathin molecular layers, operates far from the BR molecular absorption bands, which allows it to modulate near-infrared probe beams with a low-intensity visible pump [47-49]. The switching of the near-infrared wavelengths at $1310 / 1550 \mathrm{~nm}$ in single-mode optical fibers ensures that the propagation losses in the connecting fibers over short distances are negligible.

The switching contrast can be maximized by suitably choosing the number of BR-monolayers, the photoisomerization of which shifts the resonance to the midpoint between the original position and that of the nearest neighbor. It also increases with the increase in the polarizability. Switching can be achieved at very low powers $(\leq \mu \mathrm{W})$, assuming the $Q$-factor of the microsphere does not change when choosing small BR-coated microspheres as switches, since the intensity of the pump is resonantly enhanced by recirculation in the microcavity.

An ideal all-optical switch requires low switching power, high speed, and high contrast. In general, high $Q$-factor 
results in low switching power whereas smaller cavity lifetime $\tau$ ( $=Q / \omega_{0}$, where $\omega_{0}$ is the resonant frequency) and low total internal loss $A_{i}\left(=\alpha_{\text {bend }} 2 \pi \mathrm{R}\right)$, result in high speed and contrast, respectively. In our case, the high $Q$ factor of $5 \times 10^{5}$ and the high sensitivity of BR results in all-optical switching at very low powers $(<200 \mu \mathrm{W}$ for the control beam at $532 \mathrm{~nm}$ ). As mentioned earlier, the switch in our experiments was not optimized for best performance. In a BR-coated silica microcavity, the switching speed depends on BR isomerization, which in our experiment was $\sim \mu$ s and can be optimized by controlling various factors such as, by tailoring the BR intermediate lifetimes and/or phototransitions, by exploiting ultrafast photochromic transitions of the early BR intermediate states studied for switching $[52,53]$, or by coating the microcavity by other organic molecules such as diarylethenes that exhibit ultrafast photochromic transitions, in which case, the speed is limited by the cavity photon lifetime.

As mentioned earlier, the transmission spectra exhibited an extinction of $-9.4 \mathrm{~dB}$ in Port 2 and a $9.8 \mathrm{~dB}$ increase in transmission in Port 3. In our experimental conditions a $5 \mathrm{~mW}$ DFB laser operating at $1310 / 1550 \mathrm{~nm}$ was used to butt couple light into the SMF fiber. Considering $~ 30 \%$ light to couple through $(1.5 \mathrm{~mW})$ as the input signal, $\sim 98 \%$ of the light $(1.47 \mathrm{~mW})$ gets switched from Port 1 to Port 4 , or from Port 3 to Port 2, when the microcavity is activated and only $\sim 2 \%$ gets coupled into Port 2 or Port 4 respectively. Considering negligible signal loss due to scattering and propagation through the short length of the connecting optical fibers, can result in cascading of a large number of switches. For instance, considering $1.5 \mathrm{~mW}$ of coupled light in Port 1 and a terminal signal strength of $\sim 0.2 \mathrm{~mW}$ can result in principle, in cascading as many as 65 switches.

In general, the BR-coated microcavity switch is operated with outputs coded on two different wavelengths. Cascading these switches in general would require the output at one wavelength from one switch to form the input at the other wavelength for the next switch. This would then require a wavelength converter. However, as shown in the proposed designs for various Boolean arithmetic and logic circuits earlier $[49,50]$ and in the present designs of all-optical Toffoli, Peres, and Feynman Double gates, which use multiple cascaded switches, using one wavelength as the signal and the other as a control signal, circuits can be suitably designed to circumvent the use of a wavelength converter. Using microcavities as the basic building block also offers the possibility of using arrays and 2D/3D integrated structures to form circuits and networks. The design of the switch can be tailored to meet device specifications by optimizing the microcavity, by changing its shape or size, as well as the BR response, using the powerful capabilities of nanobiotechnological techniques.

An ideal photosensitive material should exhibit high sensitivity, high absorption, fast dynamics, high photo and thermal stability, and potential to tailor its properties, as coating the microcavities with a photosensitive material is of critical value. BR protein is a natural photochromic material that exhibits this unique combination of properties for practical realization. Besides its unique properties, BR is a good choice of coating a microsphere as (i) its structure is well known, (ii) it self-assembles easily onto a silica surface, (iii) its molecular configuration can be switched optically between two stable states, and (iv) a pump-probe excitation can be easily implemented. The control of all these features provides tremendous scope for tuning and optimizing the characteristics of the BR-coated microcavity switch to meet device requirements. Microcavities coated with photochromic materials such as BR have advantages in terms of simple geometry, ease of fabrication, high thermal and photostability, high fan-out, cost-effectiveness, and lowpower operation.

It is extremely important that the photochromic material exhibits high fatigue resistance for practical applications. It is remarkable that $\mathrm{BR}$ retains its properties in film form even after being heated to $140^{\circ} \mathrm{C}$ whereas in liquid solutions it has been shown to be stable upto $80^{\circ} \mathrm{C}$ [42-46]. It has been reported that $\mathrm{BR}$ films do not show any photodegradation even after $10^{6}$ switching cycles and more than 10 years of use. BR also exhibits photochemical stability over a wide $\mathrm{pH}$ range (0-12) [44]. These exceptional properties are attributed to the fact that it survives in extremely harsh conditions, has been optimized over centuries of evolution and it possesses a 2D crystalline structure.

Recently, reversible tuning of a photonic crystal cavity resonance has been demonstrated using a thin photochromic film composed of spiropyran and polymethylmethacrylate that serves as a photosensitive cladding layer [55]. This system suffers from photostability and hence repeatability as the cavity can be shifted and reversed roughly only three times before the tunability degrades too much for further significant shifts [55].

Recently, Directed Logic (DL) has been demonstrated as an innovative paradigm that minimizes the latency in calculating a complicated logic function by taking advantage of fast and low-loss propagation of light in integrated and waveguided on-chip photonic system [5]. The requirements for an ideal optical switch for DL circuits are small size, fast switching speed, low-power consumption, and capability of large scale integration. The first general designs of reversible logic gates proposed with optically controlled microresonators that can be implemented in both fiber or integrated-optic format can meet the desired specifications.

There is tremendous research underway to fabricate high-Q microring resonators in a variety of materials and large-scale arrays of ultrahigh- $Q$ coupled nanocavities [29, $31,34]$. Recently, a compact silicon ring resonator coated with vanadium dioxide $\left(\mathrm{VO}_{2}\right)$ has been demonstrated by thermally inducing the metal-insulator transition in $\mathrm{VO}_{2}$ and hence the refractive index. The proof-of-principle proposition for $\mathrm{THz}$ operation is based on $\sim 3 \mu \mathrm{m}$ diameter rings with $Q \sim 10^{2}-10^{3}$ that requires changing the temperature from room temperature to $80^{\circ} \mathrm{C}$, which is severely limited for practical applications [56]. We intend to investigate an all-optical BR-coated microring resonator switch in planar geometry for all-optical device applications that would be presented elsewhere.

The proposed designs require optical excitation of BR protein, which in principle requires some energy, which due to its high quantum efficiency, high absorption coefficient, 
and high sensitivity, is very low. Moreover, as ultra-high$Q$ microresonator cavities offer the potential for singlemolecule detection, the integration of a single BR molecule, or a few BR molecules with an ultra-high- $Q$ microresonator configuration, may possibly lead to an extremely small energy excitation of $\mathrm{BR}$ that is enough for activating the microresonator switch and enabling reversible logic operations. Hence, although the proposed designs are important, as they demonstrate the feasibility for low-power all-optical computing, the potential for single-photon implementation could lead to the energy for excitation of BR in the proposed configuration to drop below kTln2, for realizing the much desired zero-energy-dissipation logic.

\section{Conclusion}

We have presented the designs of various reversible alloptical logic gates with optically controlled microresonators, namely, the Feynman gate, Toffoli gate, Peres gate, and the Feynman double gate. To demonstrate the applicability of the designs, a basic all-optical switch has been demonstrated at telecom wavelengths, controlled with visible light, using the unique $\mathrm{BR}$ protein monolayers coated on a silica microresonator, coupled between two tapered single-mode optical fibers. Low-power switching has been shown, which is a consequence of the high- $Q$ factor, small dimensions, and photosensitivity of BR. The proposed designs can be used to implement large computing circuits and networks within an $\mathrm{mW}$ power budget. The change in the refractive index in a BR-coated microcavity switch depends on the wavelength of the control beam making the switches tunable. Since the properties of BR protein can be tailored by physical, chemical, and genetic engineering techniques, the performance can be optimized for desired applications.

The proposed designs of all-optical reversible logic gates with BR-coated microcavity can be implemented with any other photosensitive material-coated on a microresonator and in any condition in which the resonance and the coupling can be controlled externally. They can also possibly be implemented in planar geometry using integrated-optic photonic crystal micro/nanocavities. The proposed designs are general and demonstrate the applicability of using optically controlled microresonators for reversible computing applications.

\section{Acknowledgments}

S. Roy and P. Sethi are thankful to the Department of Science and Technology, Government of India, for partial support of this paper under Grant no. DST/TSG/PT/2008/04. F. Vollmer is grateful to the Max Planck Institute for the Science of Light, Erlangen, Germany, for financial support.

\section{References}

[1] S. Roy, Ed., "Special issue on optical computing, circuits, devices and systems," IET Circuits, Devices \& Systems, vol. 5, no. 2, pp. 73-75, 2011.
[2] H. J. Caulfield and S. Dolev, "Why future supercomputing requires optics," Nature Photonics, vol. 4, no. 5, pp. 261-263, 2010.

[3] D. A. B. Miller, "Are optical transistors the logical next step?" Nature Photonics, vol. 4, no. 1, pp. 3-5, 2010.

[4] J. L. O’Brien, A. Furusawa, and J. Vuckovic, "Photonic quantum technologies," Nature Photonics, vol. 3, no. 12, pp. 687695, 2009.

[5] Q. Xu and R. Soref, "Reconfigurable optical directed-logic circuits using microresonator-based optical switches," Optics Express, vol. 19, no. 6, pp. 5244-5259, 2011.

[6] R. Landauer, "Irreversibility and heat generation in the computing process," IBM Journal of Research and Development, vol. 5, no. 3, pp. 183-191, 1961.

[7] C. H. Bennett, "Logical reversibility of computation," IBM Journal of Research and Development, vol. 17, no. 6, pp. 525532, 1973.

[8] K. Maruyama, F. Nori, and V. Vedral, "The physics of Maxwell's demon and information," Reviews of Modern Physics, vol. 81, no. 1, pp. 1-22, 2009.

[9] H. J. Caulfield, L. Qian, C. S. Vikram et al., "Conservative optical logic devices: COLD," Advances in Imaging and Electron Physics, vol. 142, pp. 1-52, 2006.

[10] A. Peres, "Reversible logic and quantum computers," Physical Review A, vol. 32, no. 6, pp. 3266-3276, 1985.

[11] E. Fredkin and T. Toffoli, "Conservative logic," International Journal of Theoretical Physics, vol. 21, no. 3-4, pp. 219-253, 1982.

[12] A. Barenco, C. H. Bennett, R. Cleve et al., "Elementary gates for quantum computation," Physical Review A, vol. 52, no. 5, pp. 3457-3467, 1995.

[13] J. Shamir, H. J. Caulfield, W. Miceli, and R. J. Seymour, "Optical computing and the Fredkin Gates," Applied Optics, vol. 25, no. 10, pp. 1604-1607, 1986.

[14] G. J. Milburn, "Quantum optical Fredkin gate," Physical Review Letters, vol. 62, no. 18, pp. 2124-2127, 1989.

[15] L. Qian and H. J. Caulfield, "Abstract passive interferometers with applications to conservative logic," Optik, vol. 116, no. 8, pp. 404-408, 2005.

[16] E. Knill, R. Laflamme, and G. J. Milburn, "A scheme for efficient quantum computation with linear optics," Nature, vol. 409, no. 6816, pp. 46-52, 2001.

[17] J. Fiurášek, "Linear-optics quantum Toffoli and Fredkin gates," Physical Review A, vol. 73, no. 6, Article ID 062313, 7 pages, 2006.

[18] K. Morita, "Reversible computing and cellular automata-a survey," Theoretical Computer Science, vol. 395, no. 1, pp. 101131, 2008.

[19] H. J. Caulfield, R. A. Soref, and C. S. Vikram, "Universal reconfigurable optical logic with silicon-on-insulator resonant structures," Photonics and Nanostructures, vol. 5, no. 1, pp. 1420, 2007.

[20] X. Q. Shao, J. J. Wen, X. R. Jin, A. D. Zhu, S. Zhang, and K. H. Yeon, "Efficient scheme for implementing a Fredkin gate via resonant interaction with two-mode cavity quantum electrodynamics," Chinese Physics Letters, vol. 24, no. 10, pp. 2730-2732, 2007.

[21] A. R. Trivedi and S. Bandyopadhyay, "Single spin ToffoliFredkin logic gate," Journal of Applied Physics, vol. 103, no. 10, Article ID 104311, 5 pages, 2008

[22] D. H. Wood and J. Chen, "Fredkin gate circuits via recombination enzymes," Proceedings of the IEEE Congress on Evolutionary Computation (CEC '04), vol. 2, pp. 1896-1900, 2004. 
[23] A. J. Poustie and K. J. Blow, "Demonstration of an all-optical Fredkin gate," Optics Communications, vol. 174, no. 1-4, pp. 317-320, 2000.

[24] N. Kostinski, M. P. Fok, and P. R. Prucnal, "Experimental demonstration of an all-optical fiber-based Fredkin gate," Optics Letters, vol. 34, no. 18, pp. 2766-2768, 2009.

[25] P. Remón, R. Ferreira, J. M. Montenegro, R. Suau, E. PérezInestrosa, and U. Pischel, "Reversible molecular logic: a photophysical example of a Feynman Gate," ChemPhysChem, vol. 10, no. 12, pp. 2004-2007, 2009.

[26] T. C. Ralph, K. J. Resch, and A. Gilchrist, "Efficient Toffoli gates using qudits," Physical Review A, vol. 75, no. 2, Article ID 022313, 5 pages, 2007.

[27] S. X. Qiang, C. Li, and Z. Shou, "Two simple schemes for implementing Toffoli gate via atom-cavity field interaction in cavity quantum electrodynamics," Chinese Physics B, vol. 18, no. 2, pp. 440-445, 2009.

[28] T. Monz, K. Kim, W. Hänsel et al., "Realization of the quantum Toffoli gate with trapped ions," Physical Review Letters, vol. 102, no. 4, Article ID 040501, 2009.

[29] K. J. Vahala, "Optical microcavities," Nature, vol. 424, no. 6950, pp. 839-846, 2003.

[30] S. J. B. Yoo, "Future prospects of silicon photonics in next generation communication and computing systems," Electronics Letters, vol. 45, no. 12, pp. 584-588, 2009.

[31] L. D. Haret, T. Tanabe, E. Kuramochi, and M. Notomi, "Extremely low power optical bistability in silicon demonstrated using 1D photonic crystal nanocavity," Optics Express, vol. 17, no. 23, pp. 21108-21117, 2009.

[32] T. A. Ibrahim, R. Grover, L. C. Kuo, S. Kanakaraju, L. C. Calhoun, and P. T. Ho, "All-optical AND/NAND logic gates using semiconductor microresonators," IEEE Photonics Technology Letters, vol. 15, no. 10, pp. 1422-1424, 2003.

[33] T. C. H. Liew, A. V. Kavokin, and I. A. Shelykh, "Optical circuits based on polariton neurons in semiconductor microcavities," Physical Review Letters, vol. 101, no. 1, Article ID 016402, 4 pages, 2008.

[34] M. Notomi, E. Kuramochi, and T. Tanabe, "Large-scale arrays of ultrahigh-Q coupled nanocavities," Nature Photonics, vol. 2, no. 12, pp. 741-747, 2008.

[35] M. Trupke, J. Goldwin, B. Darquié et al., "Atom detection and photon production in a scalable, open, optical microcavity," Physical Review Letters, vol. 99, no. 6, Article ID 063601, 2007.

[36] D. K. Armani, T. J. Kippenberg, S. M. Spillane, and K. J. Vahala, "Ultra-high-Q toroid microcavity on a chip," Nature, vol. 421, no. 6926, pp. 925-928, 2003.

[37] M. Pöllinger, D. O’Shea, F. Warken, and A. Rauschenbeutel, "Ultrahigh-Q tunable whispering-gallery-mode microresonator," Physical Review Letters, vol. 103, no. 5, Article ID 053901, 2009.

[38] F. Vollmer and S. Arnold, "Whispering-gallery-mode biosensing: label-free detection down to single molecules," Nature Methods, vol. 5, no. 7, pp. 591-596, 2008.

[39] H. C. Tapalian, J. P. Laine, and P. A. Lane, "Thermooptical switches using coated microsphere resonators," IEEE Photonics Technology Letters, vol. 14, no. 8, pp. 1118-1120, 2002.

[40] A. Tewary, M. J. F. Digonnet, J. Y. Sung, J. H. Shin, and M. L. Brongersma, "Silicon-nanocrystal-coated silica microsphere thermooptical switch," IEEE Journal on Selected Topics in Quantum Electronics, vol. 12, no. 6, pp. 1476-1479, 2006.

[41] D. Oesterhelt and W. Stoeckenius, "Rhodopsin-like protein from the purple membrane of Halobacterium halobium," Nature, vol. 233, no. 39, pp. 149-152, 1979.
[42] R. R. Birge, "Photophysics and molecular electronic applications of the rhodopsins," Annual Review of Physical Chemistry, vol. 41, no. 1, pp. 683-733, 1990.

[43] D. Oesterhelt, C. Brauchle, and N. Hampp, "Bacteriorhodopsin: a biological material for information processing," Quarterly Reviews of Biophysics, vol. 24, no. 4, pp. 425-478, 1991.

[44] N. Hampp, "Bacteriorhodopsin as a photochromic retinal protein for optical memories," Chemical Reviews, vol. 100, no. 5, pp. 1755-1776, 2000.

[45] S. Roy, C. P. Singh, and K. P. J. Reddy, "Generalized model for all-optical light modulation in bacteriorhodopsin," Journal of Applied Physics, vol. 90, no. 8, pp. 3679-3688, 2001.

[46] N. Hampp and D. Oesterhelt, "Nanobiotechnology: concepts, applications and perspectives," in Nanobiotechnology, C. Niemeyer and C. Mirkin, Eds., pp. 146-167, Wiley-VCH Verlag GmbH \& Co., Weinheim, Germany, 2004.

[47] J. Topolancik and F. Vollmer, "All-optical switching in the near infrared with bacteriorhodopsin-coated microcavities," Applied Physics Letters, vol. 89, no. 18, Article ID 184103, pp. $1-3,2006$.

[48] J. Topolancik and F. Vollmer, "Photoinduced transformations in bacteriorhodopsin membrane monitored with optical microcavities," Biophysical Journal, vol. 92, no. 6, pp. 2223-2229, 2007.

[49] S. Roy, M. Prasad, J. Topolancik, and F. Vollmer, "All-optical switching with bacteriorhodopsin protein coated microcavities and its application to low power computing circuits," Journal of Applied Physics, vol. 107, no. 5, Article ID 053115, 9 pages, 2010.

[50] S. Roy and M. Prasad, "Novel proposal for all-optical Fredkin logic gate with bacteriorhodopsin coated microcavity and its applications," Optical Engineering, vol. 49, no. 6, Article ID 065201, 11 pages, 2010.

[51] C. P. Singh and S. Roy, "All-optical switching in bacteriorhodopsin based on M state dynamics and its application to photonic logic gates," Optics Communications, vol. 218, no. 13, pp. 55-66, 2003.

[52] S. Shim, J. Dasgupta, and R. A. Mathies, "Femtosecond time-resolved stimulated raman reveals the birth of bacteriorhodopsin's J and K intermediates," Journal of the American Chemical Society, vol. 131, no. 22, pp. 7592-7597, 2009.

[53] L. Fabian, E. K. Wolff, L. Oroszi, P. Ormos, and A. Der, "Fast integrated optical switching by the protein bacteriorhodopsin," Applied Physics Letters, vol. 97, no. 2, Article ID 023305, 7 pages, 2010.

[54] M. Cai, O. Painter, and K. J. Vahala, "Observation of critical coupling in a fiber taper to a silica-microsphere whisperinggallery mode system," Physical Review Letters, vol. 85, no. 1, pp. 74-77, 2000.

[55] D. Sridharan, E. Waks, G. Solomon, and J. T. Fourkas, "Reversible tuning of photonic crystal cavities using photochromic thin films," Applied Physics Letters, vol. 96, no. 15, Article ID 153303, pp. 1-3, 2010.

[56] J. Nag, J. D. Ryckman, M. T. Hertkorn, B. K. Choi, R. F. Haglund, and S. M. Weiss, "Ultrafast compact silicon-based ring resonator modulators using metal-insulator switching of vanadium dioxide," in Physics and Simulation of Optoelectronic Devices 18, vol. 7597 of Proceedings of SPIE, pp. 0-9, 2010. 

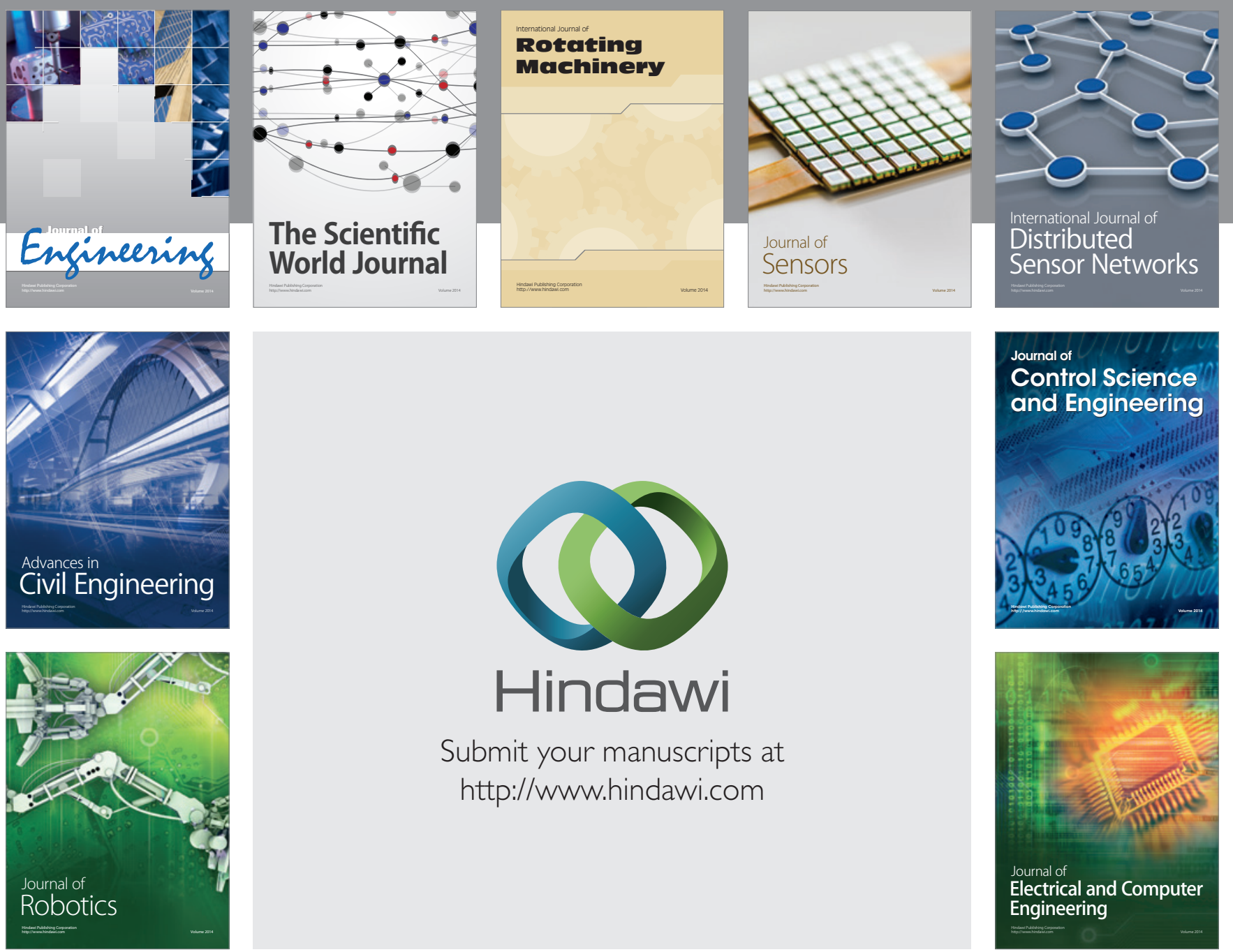

Submit your manuscripts at

http://www.hindawi.com
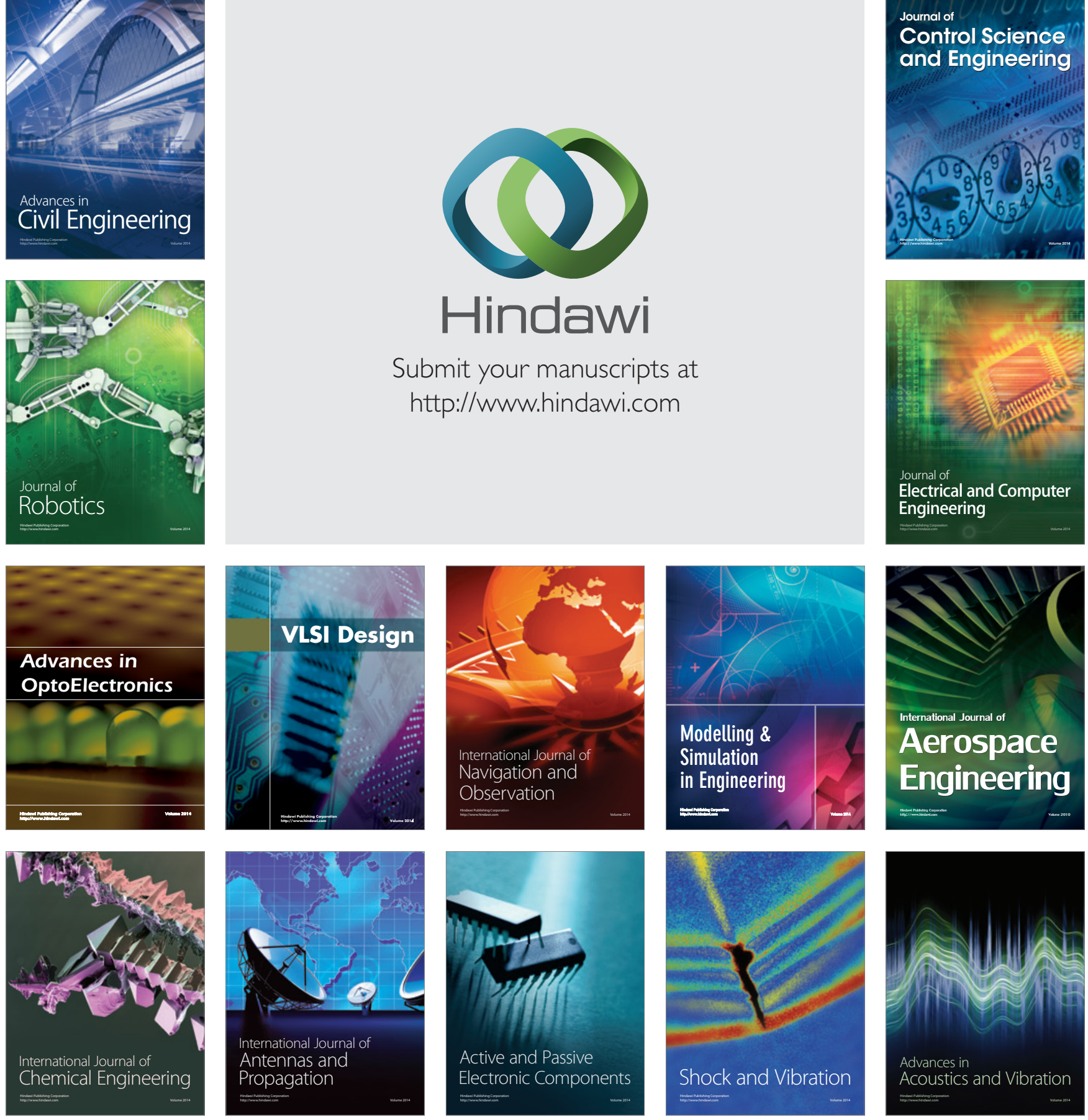